Images in...

\title{
A twist of fate with a turn of neck
}

\author{
Mubashira Hashmi, ${ }^{1}$ Shaista Siddiqi, ${ }^{2}$ Shahid Mustafa, ${ }^{1}$ Khurram Siddiqi, ${ }^{3}$ Naveeduddin Ahmed, ${ }^{2}$ \\ Naveed Ahmed, ${ }^{4}$ Farrukh Shohab ${ }^{1}$
}

${ }^{1}$ Department of Neurology, Aga Khan University and Hospital, Karachi, Pakistan
2 Department of Neurology, Liaquat National Hospital, Karachi, Pakistan
${ }^{3}$ Department of Neurology, King Fahad Medical City, Riyadh, Saudi Arabia
${ }^{4}$ Department of Radiology, Liaquat National Hospital, Karachi, Pakistan

Correspondence to Mubashira Hashmi, dr mhashmi@hotmail.com

\section{DESCRIPTION}

A 45-year-old man, presented with right-sided headache and neck pain, blurring of vision, vomiting, numbness and weakness of the right half of the body. On query, he gave a history of neck massage by professional masseurs, 2 weeks prior to the presentation, with an episode of brief loss of consciousness during the massage. On examination he was awake, alert, had symmetrical pulses and blood pressures with right hemi-paresis and hemi-anaesthesia.

Cranial MRI demonstrated ischaemic infarcts in the left cerebellar, the left occipital and the left thalamic region (figure $1 \mathrm{~A}$ ) and an intraluminal thrombus in the left vertebral artery (figure 1B). A subtracted three-dimensional CT angiography revealed dissection along posterio-superior aspect of the left subclavian artery, involving the origin of vertebral artery (figure 1C,D). This most likely resulted in multiple emboli from intravertebral thrombus, which was evident by the presence of ischaemic lesions on the side of the affected vessel. He was started on anticoagulation and discharged with almost complete recovery.

A broad spectrum of injuries has been related to cervical (carotid and vertebral) artery dissection, including chiropractic neck manipulation and use of hand-held electric neck massager. ${ }^{12}$ Subclavian artery dissection is a very rare cause of posterior circulation stroke. It is reported as idiopathic, with aortic vascular anomalies or following blunt
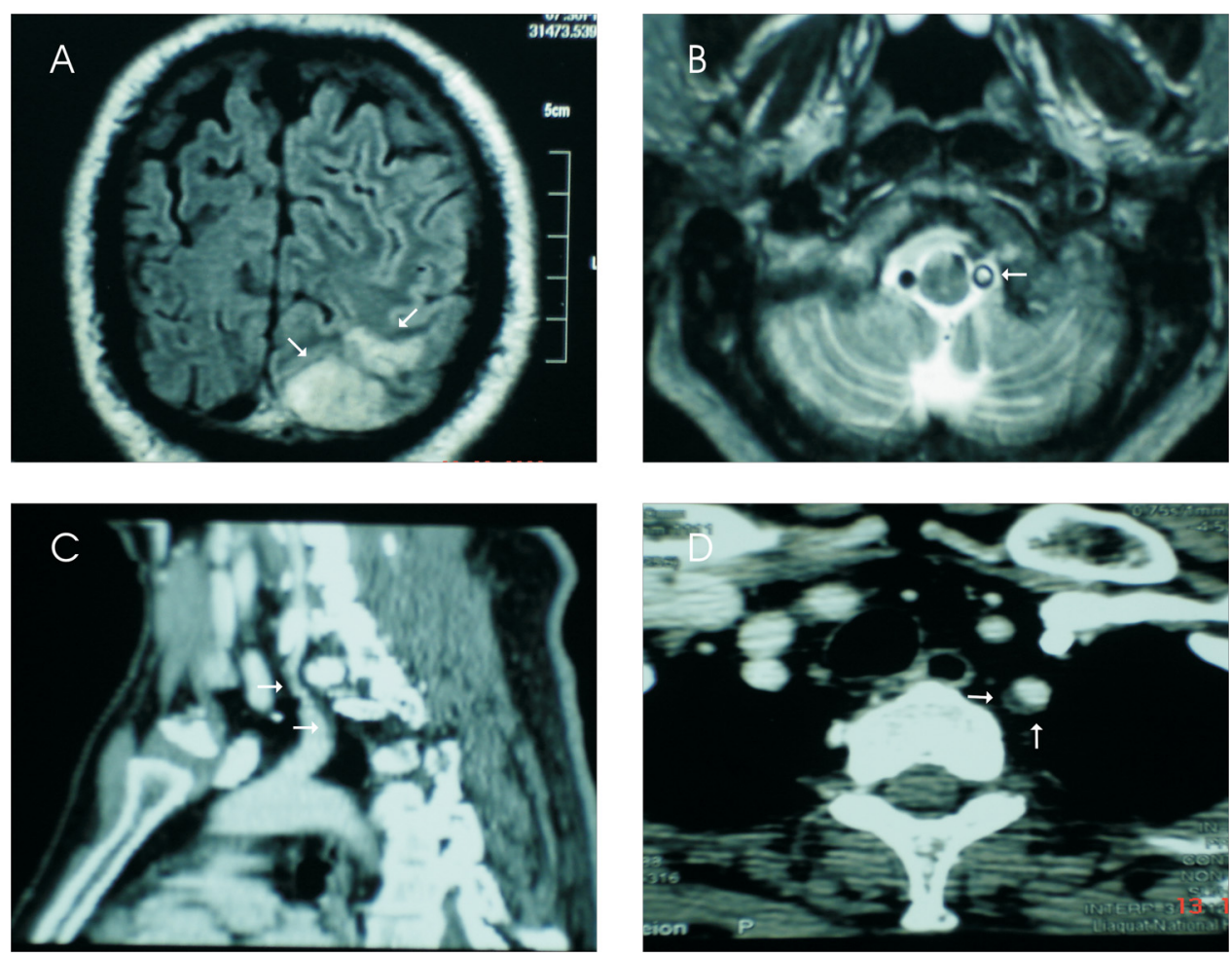

Figure $1 \mathrm{MRI}$ and three-dimensional (3D)-CT angiography findings. (A) Coronal fluid-attenuated inversion recovery MR image showing left occipital infarct (arrows). (B) Axial T-2 weighted MR image demonstrating an intraluminal thrombus in left vertebral artery (arrows). (C) 3D CT angiography showing dissection along posteriosuperior aspect of left subclavian artery, involving origin of left vertebral artery (arrows). (D) Axial view showing a narrowed eccentric lumen of left subclavian artery (arrows). 


\section{BMJ Case Reports}

neck trauma. ${ }^{3}$ To the best of our knowledge, this is the first report of subclavian artery dissection and posterior circulation stroke secondary to neck massage.

Neck massage, a frequent practice in Asian countries, can result in devastating complications including subclavian artery dissection that needs urgent recognition and management.

\section{REFERENCES}

1. Rothwell DM, Bondy SJ, Williams JI. Chiropractic manipulation and stroke. A population-based case-control study. Stroke 2001;32:1054-60.

2. Grant A, Wang N. Carotid dissection associated with a handheld electric massager. South Med J 2004;97:1262-3.

3. Garewal M, Selhorst J. Subclavian artery dissection and triple infarction of the nervous system. Arch Neurol 2005;62:1917-19.

\section{Competing interests None.}

Patient consent Obtained.

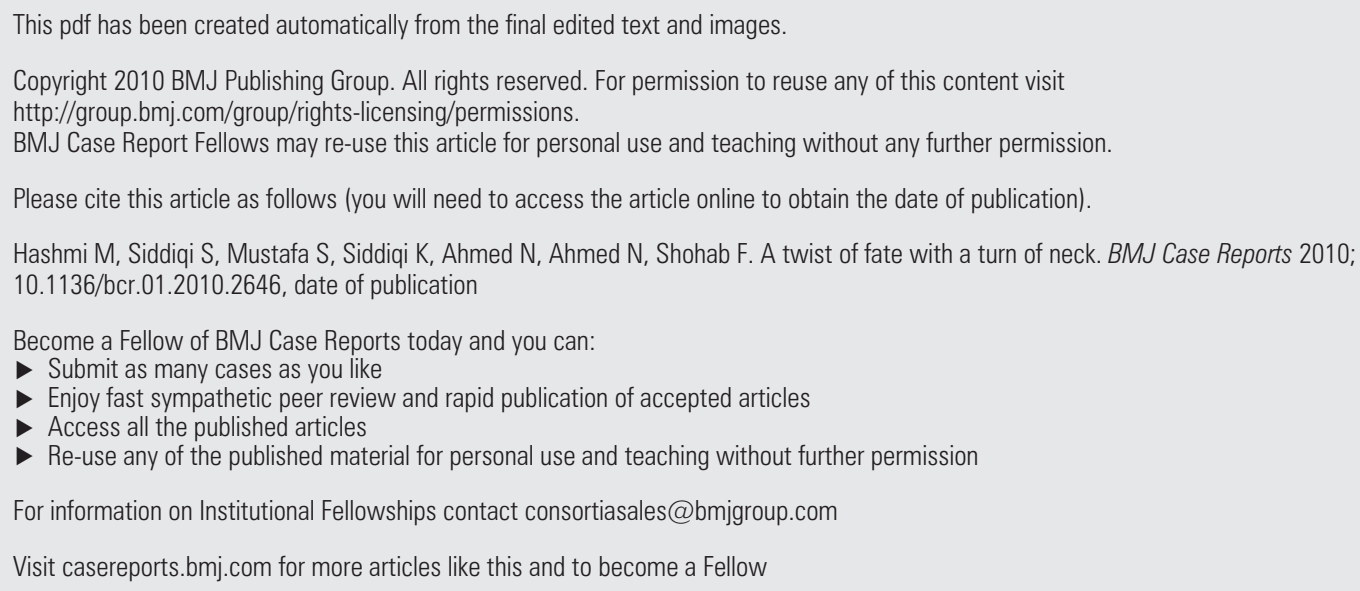

